\title{
SAGA: Um Sistema WEB para Administração e Gerenciamento remoto e em tempo real de servidores VoIP baseados no Asterisk PBX
}

\author{
Johnny Cezar Marçal dos Santos, Lindemberg Silva Pereira, Sergio Vianna Fialho
}

\begin{abstract}
Resumo-Este artigo apresenta o SAGA, um sistema WEB para administração, gerenciamento, configuração e programação on-line e em tempo real de servidores VoIP baseados no Asterisk PBX. A combinação do Asterisk PBX com o SAGA permitiu o desenvolvimento de uma solução VoIP de baixo custo, rápida e flexível, caracterizada como um servidor de aplicações VoIP com administração e configuração via WEB.
\end{abstract}

Palavras-Chave-VoIP, Asterisk PBX, WEB.

Abstract-This article presents SAGA, a WEB system for the administration, management, configuration and on-line and real time programming of VoIP servers based on Asterisk PBX. The combination of Asterisk PBX with SAGA has allowed the development of a low cost, fast and flexible new VoIP solution, characterized as a VoIP application server with WEB based administration and configuration functionalities.

Keywords - VoIP, Asterisk PBX, WEB.

\section{INTRODUÇÃO}

A cada dia mais empresas desenvolvem soluções VoIP proprietárias com diversas características e funcionalidades, geralmente agregando algum custo a esses serviços. Ainda são raras as soluções VoIP de baixo custo e/ou gratuitas.

No contexto de soluções VoIP de baixo custo, o Asterisk PBX ganha destaque: ele implementa, através de software, as funcionalidades de uma central telefônica com características e funcionalidades encontradas apenas nos modelos mais avançados de PBXs convencionais.

O SAGA é um software que foi desenvolvido para diminuir a complexidade na administração e programação do Asterisk PBX, e também para possibilitar sua administração e programação remota e em tempo real. Essas características permitem que o administrador do sistema possa, remotamente, por exemplo, adicionar e configurar um ramal para oferecer o serviço de conferência por áudio, necessitando para isso apenas de conexão com a Internet e de um navegador WEB para acessar o SAGA, ou seja, a presença física do administrador na instituição para gerenciar o ambiente VoIP não é mais obrigatória. O SAGA foi desenvolvido no PoP-RN e se baseou em trabalhos de estagiários de anos anteriores, que introduziram algumas das idéias trabalhadas [1], [2].

Johnny Cezar Marçal dos Santos (johnny@bolsistas.pop-rn.rnp.br), Lindemberg Silva Pereira (lindemberg@bolsistas.pop-rn.rnp.br), bolsistas do PoP-RN/RNP e Prof. D.Sc Sergio Vianna Fialho (fialho@pop-rn.rnp.br), coordenador do PoP-RN, professor do Departamento de Engenharia de Computação e Automação, Centro de Tecnologia, Universidade Federal do Rio Grande do Norte, Brasil. Esse projeto vem sendo financiado através do Plano de Trabalho do PoP-RN junto à RNP.
II. SAGA

O SAGA é uma ferramenta que diferencia os usuários que o acessam. Essa diferenciação é caracterizada pelas funcionalidades e informações que a ferramenta provê para cada tipo de usuário: visitante, usuário e administrador.

A ferramenta disponibiliza serviços de gerenciamento de usuários dos protocolos IAX, SIP e H323 para aplicações VoIP. Além disso, permite a administração de planos de discagens, correios de voz, filas de atendimento, conferências de voz, dentre outros. Para fins de segurança, utiliza o WEBSec, que é um módulo para prevenção, deteç̧ão e auditoria de ataques WEB, desenvolvido no escopo desse sistema.

Inicialmente foi feita a especificação formal do SAGA em UML 2.0, durante a qual foram elaborados os diagramas de casos de uso, diagrama de classes e diagramas de seqüência [1].

$\mathrm{Na}$ implementação da ferramenta foram utilizados software livres e compatíveis com a tecnologia WEB, de acordo com o apresentado na seção IV.

\section{FunCIONALIDADES}

A ferramenta oferece um conjunto de funcionalidades, que estão agrupadas em seções de acordo com o tipo de usuário. Na seção de usuário cadastrado, o mesmo pode verificar os históricos de suas últimas ligações, verificar uma estimativa de tarifação, ler notícias sobre o serviço VoIP e buscar ramais de outros usuários do sistema. A seção administrador é a mais importante do SAGA, sendo composta por um conjunto de 12 módulos. São eles: Plano de Discagem, Registro de Chamadas, LOGS, Conferência, Filas, Correio de Voz, Administradores, IAX, SIP, H323, Usuários e Segurança. Dentre os módulos, podem ser destacados: Plano de Discagem - Permite que o Servidor VoIP seja programado via WEB, habilitando ramais e novos serviços como conferências de áudio, filas de atendimento, dentre outros; Registro de Chamadas - Permite a visualização de todo histórico de ligações do Servidor, com possibilidade de aplicar filtros baseados em doze parâmetros, tais como: data, hora, tempo de chamada, usuário, aplicação, status da chamada, dentre outros; Segurança - Permite a visualização de informações relacionadas a ataques efetuados no SAGA, como: data, hora, tipo de ataque e IP do atacante. Os demais módulos estão relacionados à administração dos serviços providos pelo sistema. 


\section{Arquitetura dA SOluÇão}

A arquitetura da solução VoIP elaborada e na qual o SAGA está inserido pode ser vista na Figura 1. Cada subitem da Figura 1 possui um número de identificação e seus componentes são descritos de forma breve a seguir:

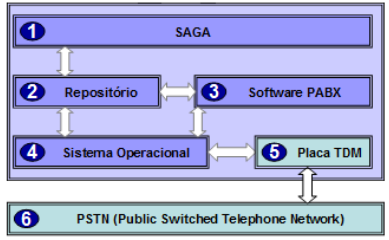

Fig. 1. Arquitetura da solução.

1. SAGA - Sistema WEB que administra e configura o Servidor VoIP. Utiliza o Servidor WEB Apache 2;

2. Repositório - Utiliza o Sistema Gerenciador de Banco de Dados MySQL 5.0 para armazenamento e recuperação de informações;

3. Software PABX - Utiliza o Asterisk PBX 1.4.8 [3], [4] para implementação dos serviços VoIP;

4. Sistema Operacional - Utiliza o Debian 4 Etch;

5. Placa TDM - Placa Digium TDM 400, que possui interfaces analógicas para comunicação com a PSTN;

6. PSTN - Utiliza ramais telefônicos da UFRN (Universidade Federal do Rio Grande do Norte);

\section{AMBIENTE VoIP DO PoP-RN}

O SAGA é uma das ferramentas que compõem o ambiente dedicado ao serviço VoIP no PoP-RN. Ele é o responsável pela administração, gerenciamento e programação de novos serviços. O ambiente VoIP pode ser observado na Figura 2. Ele permite comunicação entre chamadas via Internet, Internet para PSTN e vice-versa. Mesmo quando o destino das chamadas está atrás de um sub-rede que utiliza NAT, a comunicação ocorre de forma transparente, sem a necessidade de configurações ou servidores Proxy adicionais. Isso é possível devido à utilização do protocolo IAX2 no ambiente.

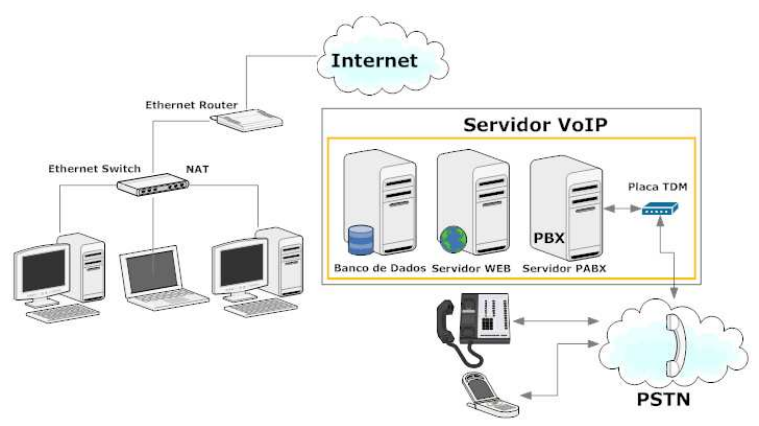

Fig. 2. Ambiente VoIP do PoP-RN.

\section{RESUltados}

O SAGA e o ambiente VoIP do PoP-RN estão em atividade a pouco mais de um ano. Nesse tempo, o SAGA se mostrou uma ferramenta bastante útil, principalmente no quesito de configuração do Servidor VoIP a distância, via WEB, e na facilidade com que permite que o administrador do sistema gerencie planos de discagem e serviços VoIP complexos, como filas de atendimento e conferências de áudio. Uma das diversas telas que compõem o SAGA pode ser vista na Figura 3. Essa interface permite que o administrador realize uma filtragem no registro de chamadas pelo tempo de duração das chamadas.

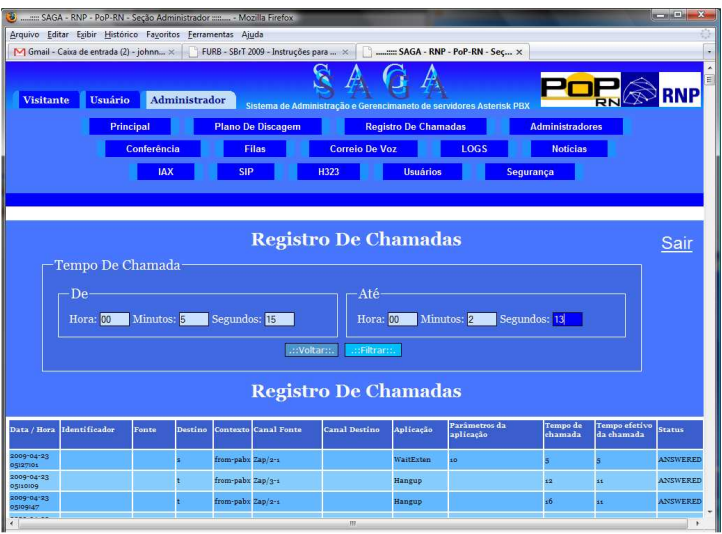

Fig. 3. Interface para filtragem das chamadas por tempo de duração.

Em breve, o SAGA será encapsulado em uma licença pública, como a GPL (General Public License), e fará parte do mundo do software livre, podendo assim, ser utilizado de forma gratuita.

\section{CONCLUSÕES}

O Asterisk PBX é um programa extremamente robusto, confiável e flexível. Mas também é um software grande e complexo, que geralmente é configurado e programado através de linhas de comandos, interfaces textuais e arquivos de texto puro. Logo, se mostrou bastante conveniente a utilização de uma ferramenta com interface gráfica como o SAGA, que tornou a configuração e programação do Asterisk PBX mais amigável para o administrador. Além disso, a característica do SAGA que permite acesso ao servidor via WEB facilitou bastante a configuração remota do serviço, uma vez que o Asterisk PBX é instalado no servidor e, exigiria a presença física do administrador no terminal de comandos desse software para realizar alterações.

A combinação do Asterisk PBX com o SAGA permitiu o desenvolvimento de uma nova solução VoIP de baixo custo, rápida, segura e flexível. Essa é, sem dúvida, uma solução viável para empresas com poucos recursos e grande necessidade de comunicação.

\section{REFERÊNCIAS}

[1] Danilo José Araújo da Silva, Desenvolvimento Inicial de um Servidor VoIP Baseado em Tecnologia WEB, Trabalho de conclusão de curso, UFRN, Natal-RN, Novembro de 2007.

[2] Higor Campos Dias Gondim, Implantação do ambiente VoIP no PoP$R N$, Relatório técnico PoP-RN, Natal-RN, Agosto de 2008.

[3] Jim Van Meggelen, Jared Smith, Leif Madsen, Asterisk - The Future Of The Telephony, Editora O'Reilly, 2007 - ISBN 10: 0-596-51048-9.

[4] "Asterisk", http://www.digium.com. 